\title{
ATMOSPHERIC SCATTERING EFFECTS ON GROUND- BASED FABRY-PEROT MEASUREMENTS OF THERMOSPHERIC WINDS: AN INVERSION TECHNIQUE
}

\author{
VINCENT J. ABREU \\ Space Physics Research Laboratory, The University of Michigan, Ann Arbor, MI 48109, U.S.A.
}

(Received 20 March 1985)

\begin{abstract}
The theoretical development of a technique to recover velocities measured with a Fabry-Perot interferometer in the presence of scattered light is presented. Simulations are carried out which show that the inversion is effective in recovering actual velocities, especially in instances when the observed brightness is dominated by scattered light.
\end{abstract}

\section{INTRODUCTION}

Ground-based Fabry-Perot interferometers are widely used to measure the Doppler shift of thermospheric emission lines. Wind velocities are deduced from these measurements under the assumption that the source of the observed brightness consists solely of non-scattered light. Abreu et al. (1983) have recently shown that scattering by tropospheric aerosols is a possible source of contamination of the measured brightness. Their simulations show that in the presence of emission gradients and a scattering atmosphere the deduced wind velocities may not represent the actual thermospheric wind and that, in fact, these factors can be responsible for diverging or converging patterns sometimes observed in the wind field deduced from Fabry-Perot ground-based measurements. They presented a concrete example of this phenomenon using the wind field pattern deduced from observations made at the Michigan Airglow Observatory (MAO) site at Calgary, Alberta (51.08 N, 114.1 W). Another point of importance brought out by their simulations is the fact that scattering may affect the measured vertical line-of-sight speeds, which may be as high as a few tens of meters per seconds in situations of large intensity gradients such as those often found in the aurora. This is of importance to investigators who use the individual zenith measurements as a reference.

The importance of scattering for a given wind measurement must be evaluated against the known meteorological conditions and the expected aerosol distribution. The latter is very irregular in space and time although it can be monitored. In this paper the solution to the following problem is addressed : Given the optical properties of a scattering atmosphere, how can one recover the actual wind velocity from FabryPerot ground-based measurements of the Doppler shift of thermospheric emission lines? The theoretical development in the following section leads to a recovery technique which is then investigated.

\section{THEORY}

The brightness, $B_{m}(\lambda, \theta)$, measured when observing an emission layer in the presence of a scattering atmosphere consists of contributions from the direct emission and from the scattered light. Assuming that the brightness varies in only one direction, i.e., with latitude, it can be represented by

$$
B_{m}(\lambda, \theta)=\frac{B_{a}(\lambda, \theta) \mathrm{e}^{-\tau(\lambda) \sec \theta}}{\cos \theta}+B_{s}(\lambda, \theta)
$$

where $B_{a}(\lambda, \theta)$ is the brightness in Rayleighs of a thin emission layer at wavelength $\lambda, \tau(\lambda)$ is the optical depth of the atmosphere, $\theta$ is the angle of observation measured fromthe zenith, and $B_{s}(\lambda, \theta)$ is the scattering component of the observed brightness. The scattered brightness is given by

$$
B_{s}(\lambda, \theta)=\int_{-\pi / 2}^{\pi / 2} B_{a}\left(\lambda, \theta_{0}\right) W\left(\lambda, \alpha, \theta, \theta_{0}\right) \mathrm{d} \theta_{0},
$$

where

$$
W\left(\lambda, \alpha, \theta, \theta_{0}\right)=\frac{\cos \theta_{0} \sin \theta_{0}}{\pi} \int_{0}^{\pi} \sigma\left(\lambda, \alpha, \theta, \theta_{0}, \phi_{0}\right) \mathrm{d} \phi_{0},
$$

$\theta_{0}$ is the angle between an incident ray and the normal to the surface, $\alpha$ is the ground albedo and $\sigma\left(\lambda, \alpha, \theta, \theta_{0}, \phi\right)$ is the scattering transmission coefficient (assumed to be known). 
Equation (1) is a Fredholm integral equation of the second kind, which can be solved for the actual brightness, $B_{a}(\theta)$. The solution of this equation can be achieved by a Fourier expansion of $B_{m}(\theta), B_{g}(\theta)$ and $W\left(\theta, \theta_{0}\right)$. The equation then is transformed into a matrix equation of the form

$$
\hat{a}=(\hat{\mathfrak{T}}+\hat{c})^{-1} \hat{b}
$$

where $\hat{a}$ and $\hat{b}$ are column matrices containing the Fourier coefficients of $B_{a}$ and $B_{m}$, respectively, and $\hat{c}$ is an $n \times m$ matrix containing the Fourier coefficients of the expansion of the kernel, $W\left(\theta, \theta_{0}\right)$, with respect to $\theta$ and $\theta_{0}$. The identity matrix is represented by $\hat{1}$.

The inversion of equation (1) in order to obtain $B_{\mathrm{g}}(\theta)$ can be considered the first step in the velocity inversion scheme. $B_{d}(\theta)$ represents the spatial variation of the wavelength integrated brightness in an emission line. It can also be assumed that the zenith angle dependence of the intensity is the same in any wavelength band which is a fraction of the full width of the line. Thus, the observed brightness at any zenith angle and wavelength interval would depend on the spatial variation of the intensity of the emission and on the Doppler shift of the emission line. Consequently, because of a priori knowledge of the spatial variation of the intensity of the emission, the spatial variation of the Doppler shift could be determined. The presence of scattering does not affect the above arguments since the scattering mechanism in practice will not change the wavelength of the emission. Thus intuitively, we expect to be able to recover the wind velocity $V(\theta)$ from measured Fabry-Perot emission lines in the presence of atmospheric scattering.

The wavelength shift, $\Delta \lambda$, in an emission line due to a relative velocity $\mathbf{V}$, between the emitting particle and the observing station is

$$
\Delta \lambda=\frac{\lambda_{0}|\mathbf{V}|}{c} \sin \theta
$$

where $\lambda_{0}$ is the unshifted wavelength and $c$ is the speed of light. In the absence of scattering, the observed line shape, $G(\lambda, \theta)$, would be a gaussian whose width is a function of the source temperature and whose central wavelength is determined by the wind velocity according to equation (4). The observed line shape, $H(\lambda, \theta)$, in the presence of scattering, is given by

$$
\begin{aligned}
H(\lambda, \theta)= & \frac{1}{\sqrt{\pi \beta}} \frac{B_{a}(\theta)}{\cos \theta} \mathrm{e}^{-\tau(\lambda) \sec \theta} \mathrm{e}^{-\left[\lambda-\lambda^{\prime}(\theta)\right]^{2} / \beta}+\frac{1}{\sqrt{\pi \beta}} \\
& \times \int_{-\pi / 2}^{\pi / 2} B_{a}\left(\theta_{0}\right) W\left(\alpha, \theta, \theta_{0}\right) \mathrm{e}^{-\left[\lambda-\lambda^{\prime}\left(\theta_{0}\right]^{2} / \beta\right.} \mathrm{d} \theta_{0}
\end{aligned}
$$

where

$$
\begin{aligned}
\lambda^{\prime}(\theta) & =\lambda_{0}+\frac{|\mathrm{V}|}{c} \lambda_{0} \sin \theta \\
\beta^{1 / 2} & =4.3 \times 10^{-7} \lambda_{0}\left(\frac{T}{M}\right)^{1 / 2} \\
T & =\text { source temperature }(\mathrm{K}), \\
M & =\text { molecular weight of the emitting species. }
\end{aligned}
$$

The goal is to solve equation (5) for $V(\theta)$. In order to obtain a solution it is assumed that:

1. The inversion for $B_{q}(\theta)$ has been effected,

2. The wavelength bandwidth under consideration is small compared to its Doppler width, i.e.,

$\mathrm{e}^{-\left[\lambda-\lambda^{\prime}(\theta)\right]^{2} / \beta(\theta)} \cong \mathrm{e}^{-\left(\lambda-\lambda_{0}\right)^{2} / \beta(\theta)}$

$$
\times\left[1+\frac{2\left(\lambda-\lambda_{0}\right)}{\beta(\theta)} \frac{\lambda_{0} V(\theta)}{c} \sin \theta\right]
$$

3. The measured line shape, $H(\lambda, \theta)$, can be least square fitted to a gaussian function :

$$
H(\lambda, \theta)=\frac{A(\theta)}{\sqrt{\pi \beta^{\prime}(\theta)}} \mathrm{e}^{-\left[\lambda-\lambda_{a}^{*}(\theta)\right]^{2} / \beta^{\prime}(\theta)},
$$

where

$$
\lambda_{a}^{\prime}(\theta)=\lambda_{0}+\frac{\lambda_{0} V^{\prime}(\theta)}{c} \sin \theta,
$$

and $V^{\prime}(\theta), \beta(\theta)$ and $A(\theta)$ are the measured velocity, line width and brightness respectively.

4. The fitted $\beta(\theta)$ is approximately equal to the actual width of the source, i.e.,

$$
\beta^{\prime}(\theta) \simeq \beta(\theta) .
$$

The consideration of the above assumptions allows one to write equation (5) as:

$$
\begin{aligned}
& \frac{A(\theta)}{\sqrt{\pi \beta^{\prime}(\theta)}} \mathrm{e}^{-\left(\lambda-\lambda_{0}\right)^{2} / \beta^{\prime}(\theta)}\left[1+\frac{2\left(\lambda-\lambda_{0}\right) \lambda_{0}}{c \beta^{\prime}(\theta)} V^{\prime}(\theta) \sin \theta\right] \\
& =\frac{1}{\sqrt{\pi \beta^{\prime}(\theta)}} \frac{B_{a}(\theta)}{\cos \theta} \mathrm{e}^{-\tau \sec \theta} \mathrm{e}^{-\left(\lambda-\lambda_{0}\right)^{2} / \beta^{\prime}(\theta)} \\
& \times\left[1+\frac{2\left(\lambda-\lambda_{0}\right) \lambda_{0}}{c \beta^{\prime}(\theta)} V(\theta) \sin \theta\right]+\frac{1}{\sqrt{\pi}} \\
& \times \int_{-\pi / 2}^{\pi / 2} \frac{B_{a}\left(\theta_{0}\right)}{\sqrt{\beta^{\prime}(\theta)}} W\left(\theta, \theta_{0}\right) \mathrm{e}^{-\left(\lambda-\lambda_{0}\right)^{2} / \beta^{\prime}\left(\theta_{0}\right)} \\
& \times\left[1+\frac{2\left(\lambda-\lambda_{0}\right) \lambda_{0}}{c \beta^{\prime}\left(\theta_{0}\right)} V\left(\theta_{0}\right) \sin \theta_{0}\right] \mathrm{d} \theta_{0} .
\end{aligned}
$$


If one further assumes that $\beta^{\prime}(\theta)$ is not a very sensitive function of $\theta$, the exponential term in equation (6) can be taken out of the integral and cancelled throughout the equation. $A$ priori knowledge of the relation [equation (1)]:

$$
A(\theta)=\frac{B_{a}(\theta)}{\cos \theta} \mathrm{e}^{-\tau \sec \theta}+\int_{-\pi / 2}^{\pi / 2} B_{a}(\theta) W\left(\theta, \theta_{0}\right) \mathrm{d} \theta_{0}
$$

allows one to write

$$
\begin{aligned}
& A(\theta) V^{\prime}(\theta) \sin \theta=\frac{B_{a}(\theta)}{\cos \theta} \mathrm{e}^{-\mathrm{r} \sec \theta} V(\theta) \\
& \times \sin \theta+\int_{-\pi / 2}^{\pi / 2} B_{a}\left(\theta_{0}\right) W\left(\theta, \theta_{0}\right) V\left(\theta_{0}\right) \sin \theta_{0} \mathrm{~d} \theta_{0} .
\end{aligned}
$$

Equation (8) is a Fredholm integral equation of the second kind which can be solved for $V(\theta)$ in the same fashion as equation (1) was solved for $B_{a}(\theta)$.

\section{NUMERICAL SIMULATIONS}

In this section we demonstrate the validity of the inversion technique just presented. The simulation program first calculates the measured functions $B_{m}(\theta)$ and $V^{\prime}(\theta)$ from $B_{a}(\theta)$ and $V(\theta)$, which represent the actual brightness and thermospheric velocity functions, respectively. The performance of the inversion technique is evaluated by solving the reverse problem, i.e., given $B_{m}(\theta)$ and $V^{\prime}(\theta)$, equations (1) and (8) are solved for the actual functions.

The evaluation of the kernel function in the integral equations requires knowledge of the scattering transmission coefficient. The latter has been obtained by an approximate solution of the radiative transfer equation using the method of directional averaging of the radiation intensity (Sobolev, 1975; Abreu, 1983). The simulations that follow have assumed a wavelength at $6300 \AA$, an optical depth equal to 0.27 , and a measured Mie phase function (Sobolev, 1963). The optical depth value chosen is from a tabulation by Elterman (1968) for an elevation of sea level. We have also assumed a source temperature of $1500 \mathrm{~K}$. The height of the source function was taken at $250 \mathrm{~km}$, and the ground albedo was chosen equal to 0.8 , representing a snow covered ground.

The recovery process was carried out for two source functions, which are shown in Figs. 1 and 2. Each figure shows source functions $B_{a}(\theta)$ and $V(\theta)$, as well as the simulated measured brightness $B_{m}(\theta)$ and the meridional velocity, $V^{\prime}(\theta)$, inferred from the Doppler measurement. The source brightnesses have been normalized to their maximum value, i.e., $B(\theta)=S(\theta) / S(\theta)_{\max }$, where $S(\theta)$ here represents the

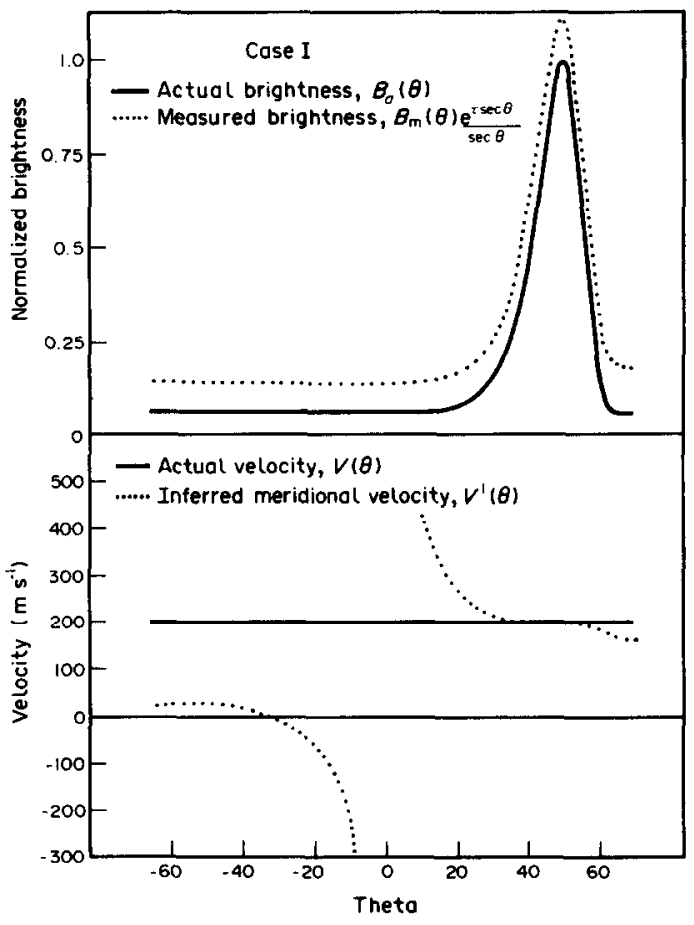

Fig. 1. (a) SOURCE FUNCTION, $B_{a}(\theta)$, REPRESENTING STRONG INTENSITY GRADIENT TO THE NORTH OF THE OBSER VING STATION AND SIMULATED MEASURED BRIGHTNESS, $\boldsymbol{B}_{m}(\theta)$; (b) ACTUAL VELOCITY, $V(\theta)$ AND MERIDIONAL VELOCITY, $V^{\prime}(\theta)$, INFERRED FROM THE SIMULATED DOPPLER MEASUREMENT.

unnormalized signal. The brightness functions are representative of auroral brightness distributions observed at the Michigan Airglow Observatory (MAO) site in Calgary, Alberta (Abreu, 1983). They represent situations in which strong intensity gradients are present to the North and near the zenith of the observing station. In the latter case, as shown in Fig. 2, a diverging wind pattern is inferred from the Doppler measurements. The recovery of $B_{a}(\theta)$ and $V(\theta)$ was effected using the Fourier inversion technique on simulated measured data in the absence of noise. The recoveries under these circumstances were near exact.

\section{ERROR ANALYSIS}

Next, we consider the effect that random errors in the measured quantities may have on the solution of the integral equations. Solving a Fredholm equation of the second kind is a well-posed mathematical problem in the sense that small uncertainties in the measured quantities do not lead to arbitrarily large errors in the solution. It is important, however, to determine how 


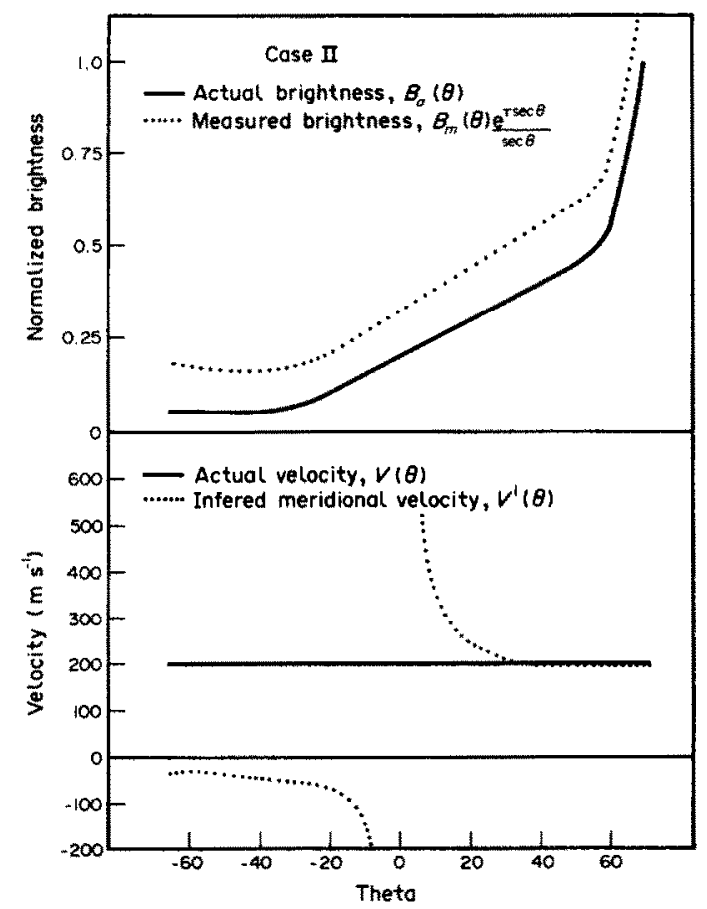

Fig. 2. (a) SOURCE FUNCTION, $B_{a}(\theta)$, REPRESENTING STRONG INTENSITY GRADIENT NEAR THE ZENITH OF THE OBSERVING STATION AND SIMULATED MEASURED BRIGHTNESS, $B_{m}(\theta) ;(b)$ ACTUAL VELOCITY, $V(\theta)$, AND MERIDIONAL VELOCITY, $V^{\prime}(\theta)$, INFERRED FROM THE SIMULATED DOPPLER MEASUREMENT.

large can the signal to noise ratio in the brightness measurements be before the inversion becomes inconsequential in improving the accuracy with which the actual brightnesses and velocities can be measured.

In order to simulate the effect of statistical errors on the inversion, gaussian noise has been added to the signal using a random number generator. The measured normalized brightness with statistical noise, $\tilde{B}_{m}(\theta)$, has been assumed to be given by

$$
\tilde{B}_{m}(\theta)=B_{m}(\theta)+B_{m}(\theta) k R
$$

where $R$ is a random number $(-1 \leqslant R \leqslant 1)$, and $k$ is the fractional deviation of the maximum photometric signal, i.e., $k=1 / \sqrt{[S(\theta)]_{\max }}$. Note that the above definition implies a constant signal to noise throughout a meridian scan. The standard deviation in the velocity measurement is dependent on the photometric brightness and is given by

$$
\sqrt{\delta \bar{V}^{2}}=\frac{\Delta \lambda \cdot \varepsilon}{\sqrt{S}}
$$

where $\Delta \lambda$ is the thermal width of the emitting source in velocity units, $\varepsilon$ is a parameter dependent on instrumental characteristics, and $S$ is the photometric counts that would be observed without the high resolution element. The parameter $\varepsilon$ varies with instrumental design and depends upon the etalon plates reflectivity, the detector and the ratio of the signal thermal width to the Fabry-Perot free spectral range. For an instrument similar to the one at the MAO in Calgary, $\varepsilon$ is approx. 2.0 and thus for a thermal width corresponding to $1500 \mathrm{~K}, \sqrt{\delta V^{2}} \simeq 1000 / \sqrt{S}$. The measured velocity with statistical noise is then given by

$$
\tilde{V}^{\prime}(\theta)=V^{\prime}(\theta)+1000 k R
$$

Equations $(9)$ and $(10)$ have been used to obtain $\tilde{B}_{m}(\theta)$ and $\tilde{V}^{\prime}(\theta)$ for the two brightness distributions shown in Figs. 1 and 2. Different values of $k$ have been used in order to obtain a signal to noise ranging from 1 to approx. 100. The Fourier inversion has then been carried out for these data in order to subtract scattering effects. The accuracy of the inversion has been expressed in terms of the fractional deviation of the recovered values from the actual. This process has been repeated for twenty sets of random numbers. The resulting average fractional deviations are shown in Figs. 3 and 4 for the brightness distribution shown in Fig. 1 for angles of observation at $-45^{\circ}$ and $+45^{\circ}$, respectively. The abscissa is the signal to noise ratio of the photometric brightness. These figures also show the average fractional deviation of the measured brightness, $\tilde{B}_{m}$, for comparison purposes (case when no inversion is effected). For either angle it can be observed that when the signal to noise is approx. 10 or greater, the inversion that takes into account scattering effects results in a more accurate recovering of the actual brightness. The inversion is most effective when one looks toward the South $\left(-45^{\circ}\right)$, where the observed emission is most contaminated by scattered light. Figures 5 and 6 show the fractional deviation of the recovered velocities from the actual for the two directions, respectively. The abscissa here is the same as before, i.e., the signal to noise ratio of the photometric brightness. The fractional deviation of the inferred meridional velocities when no inversion is carried out is also shown for comparison purposes. Figure 5 shows that when one looks directly into the auroral $\operatorname{arc}\left(+45^{\circ}\right)$ little is gained by carrying out the inversion. In Fig. 6 , where scattering effects dominate, it is apparent that for signal to noise ratios greater than 10 the inversion recovers the velocities more accurately. Figures 7 and 8 show similar results for the brightness distribution shown in Fig. 2.

The optical properties of the atmosphere under 


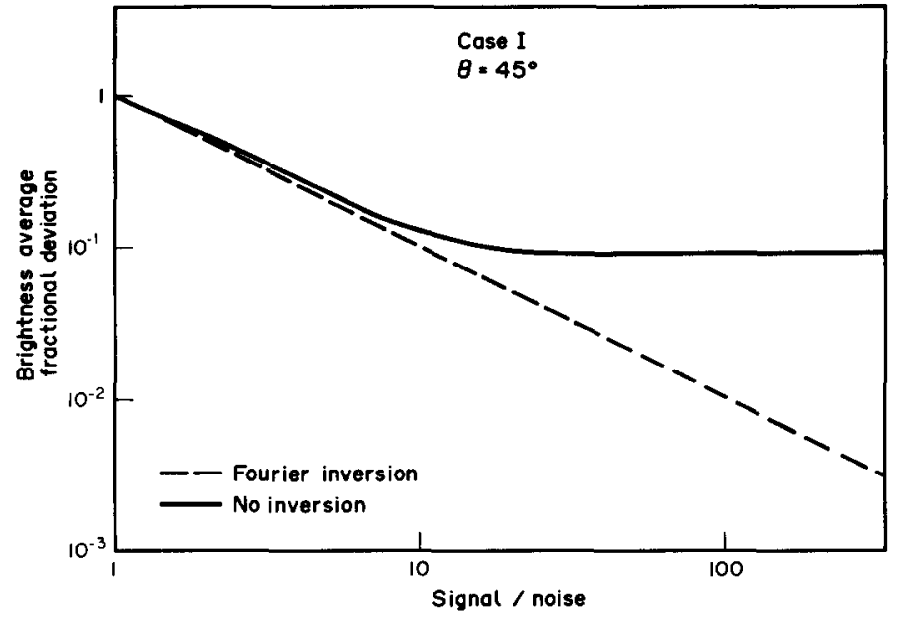

Fig. 3. Fractional deVIATION OF THE bRightNESS DISTRIBUTION SHOWN IN Fig. 1 at a ZENITH ANGLE gQUAL to $+45^{\circ}$.

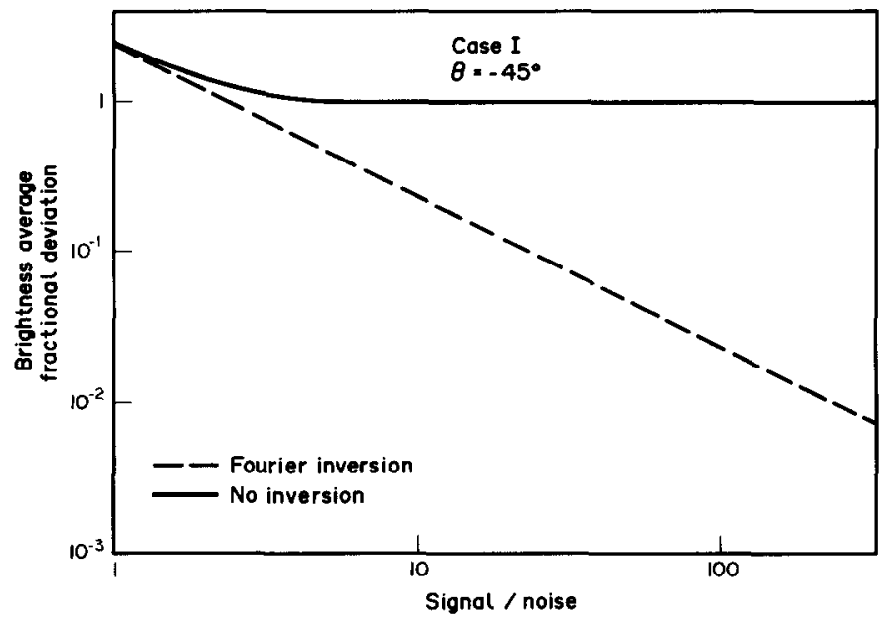

Fig. 4. Fractional deVIATION FOR THE BRIGHTNESS DISTRIBUTION SHOWN IN Fig. 1 AT A ZENITH ANGLE EQUAL TO $-45^{\circ}$.

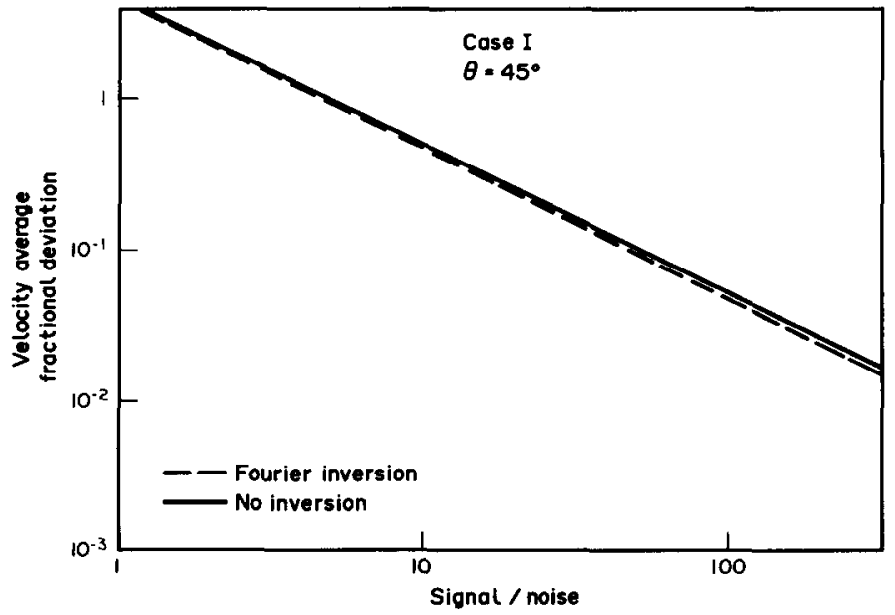

Fig. 5. FrACTIONAL DEVIATION OF THE RECOVERED VELOCITY FOR THE BRIGHTNESS DISTRIBUTION SHOWN IN FIG. 1 AT A ZENITH ANGLE EQUAL TO $+45^{\circ}$. 


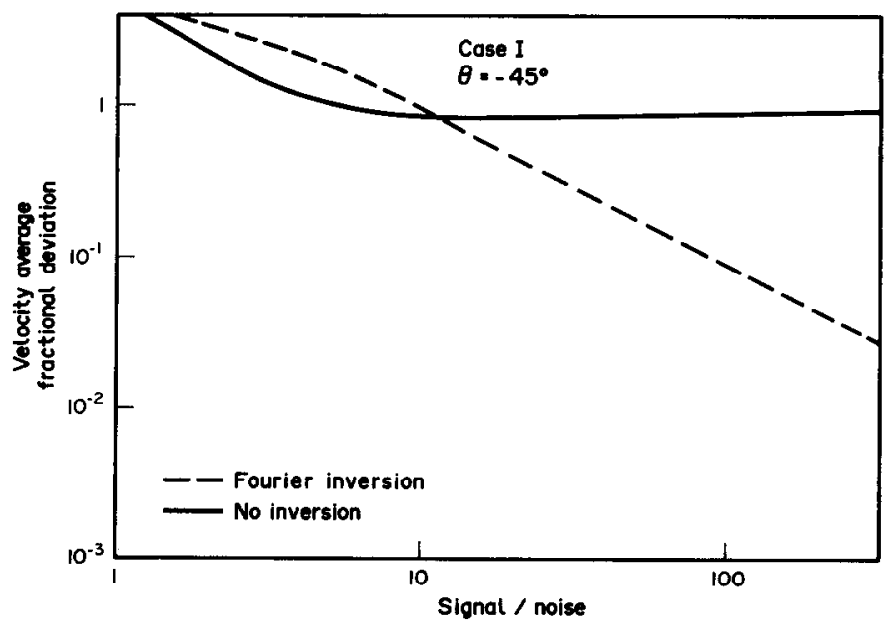

Fig. 6. FRACTIONAL DEVIATION OF THE RECOVERED VELOCITY FOR THE BRIGHTNESS DISTRIBUTION SHOWN IN FIG. 1 AT A ZENITH ANGLE EQUAL TO $-45^{\circ}$.

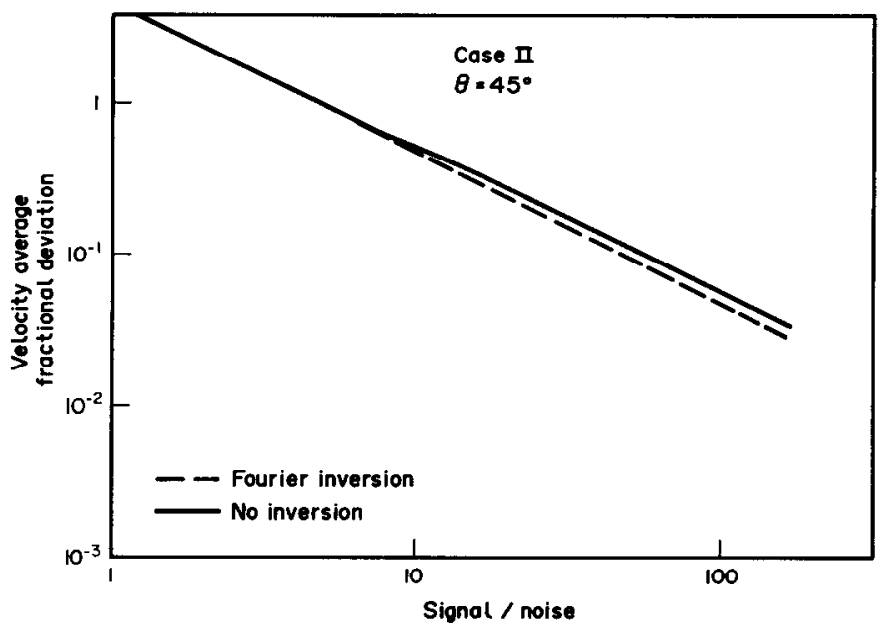

FIG. 7. FRACTIONAL DEVIATION OF THE RECOVERED VELOCITY FOR THE BRIGHTNESS DISTRIBUTION SHOWNIN FIG. 2 AT A ZENITH ANGLE EQUAL TO $+45^{\circ}$.

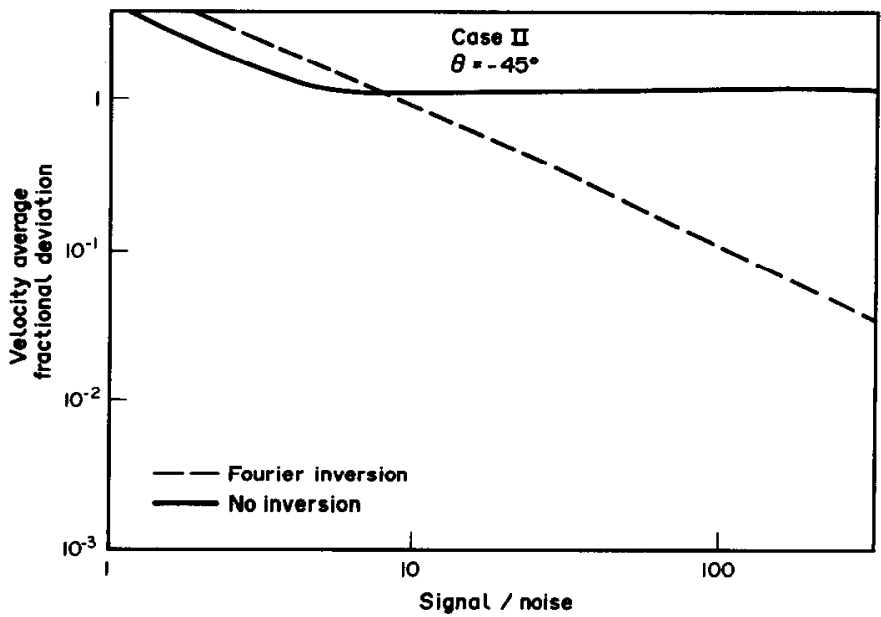

FIG. 8. FRACTIONAL DEVIATION OF THE RECOVERED VELOCITY FOR THE BRIGHTNESS DISTRIBUTION SHOWN IN FIG. 2 AT A ZENITH ANGLE EQUAL $\mathrm{mo}-45^{\circ}$. 


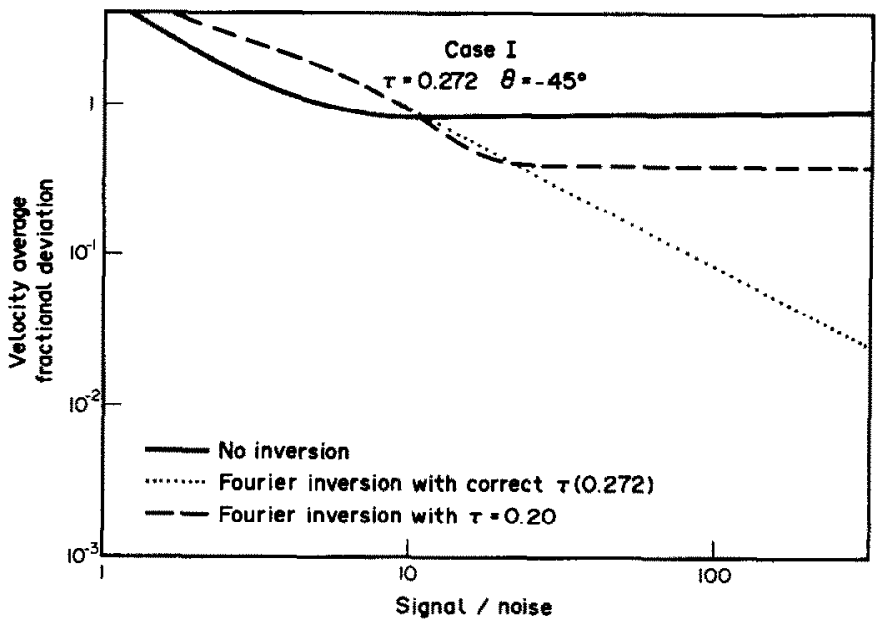

FIG. 9. VELOCITY FRACTIONAL DEVIATION FOR THE CASES WHEN THE VELOCITY IS INFERRED WITH : NO INVERSION; FOURIER INVERSION WITH CORRECT $\tau(0.272)$ AND FOURIER INVERSION WITH WRONG $\tau(0.200)$.

normal Fabry-Perot observations are not closely monitored and, consequently, large uncertainties exist in the knowledge of the optical depth and phase function associated with a given particle size distribution. It is appropriate then to estimate the error when the inversion is effected using the wrong optical depth. For this purpose, a simulation has been carried out in which $\tau=0.272$ is used in the forward calculations, i.e., in determining the measured brightness and velocities. Then a value of $\tau$ equal to 0.20 is used when the Fourier inversion is effected. The results are shown in Fig. 9. Here we show the velocity average fractional deviation for the cases when the velocity is inferred with : (a) no inversion, (b) Fourier inversion with correct $\tau(0.272)$ and (c) Fourier inversion with wrong $\tau(0.200)$. Even though the value of $\tau$ may depart from the actual by as much as $25 \%$, the recovered velocity using the Fourier inversion yields values which are closer to the actual velocity than if no inversion were effected.

\section{CONCLUSION}

The theoretical development of a technique to recover velocities measured with a Fabry-Perot interferometer in the presence of scattered light has been presented. This technique assumes knowledge of the optical properties of the atmosphere. Simulations have been carried out which show that the Fourier inversion technique is effective in recovering the actual velocities, especially in instances when the observed brightness is dominated by scattered light. It has been shown that a minimum signal to noise ratio of approx. 10 is necessary for the inversion to improve the accuracy with which the actual brightness and velocity can be determined. The effect on the recovery of not accurately knowing the atmosphere's optical depth has been investigated. It has been shown that if $\tau$ is known to within $25 \%$ of its actual value, the Fourier inversion recovery yields velocities which are closer to the actual than if no inversion were effected.

Acknowledgements-This work was supported by NSF Grant ATM-8218486.

\section{REFERENCES}

Abreu, V. J., Schmitt, G. A., Hays, P. B., Meriwether, J. W. and Tepley, C. A. (1983) Atmospheric scattering effects on ground-based measurements of thermospheric winds. Plantet. Space Sci. 31, 303.

Elterman, L. (1968) UV, visible and IR attenuation for attitudes to $50 \mathrm{~km}$. Report AFCRL-68-0153, AFCRL, Bedford, Mass.

Sobolev, V. V. (1963) Treatise on Radiative Transfer. Van Nostrand, Princeton, New Jersey.

Sobolev, V. V. (1975) Scattering in Planetary Atmospheres. Pergamon Press, Oxford. 